\title{
Acronyms and Glossary
}

Acronyms

ACIN Asociación de Cabildos Indígenas del Norte del Cauca (Association of Indigenous Cabildos of Northern Cauca)

AICO Autoridades Indígenas de Colombia (Indigenous Authorities of Colombia)

ANC Asamblea Nacional Constituyente (National Constituent Assembly)

ANUC Asociación Nacional de Usuarios Campesinos (National Association of Peasants)

ASI

Alianza Social Indígena (Indigenous Social Alliance)

AUC Autodefensas Unidas de Colombia (United Self-Defense Forces of Colombia)

CINEP Centro de Investigación y Educación Popular (Center for Research and Popular Education)

CRIC Consejo Regional Indígena del Cauca (Regional Indigenous Council of Cauca)

CRIHU Consejo Regional Indígena de Huila (Regional Indigenous Council of Huila)

DAI División de Asuntos Indígenas (Division of Indigenous Affairs)

DANE Departamento Administrativo Nacional de Estadística (National Administrative Department of Statistics)

ELN Ejército de Liberación Nacional (National Liberation Army) ETI Entidad Territorial Indígena (Indigenous Territorial Entity) 


\begin{tabular}{|c|c|}
\hline FARC & $\begin{array}{l}\text { Fuerzas Armadas Revolucionarias de Colombia } \\
\text { (Revolutionary Armed Forces of Colombia) }\end{array}$ \\
\hline INCORA & $\begin{array}{l}\text { Instituto Colombiano de la Reforma Agraria (Colombian } \\
\text { Institute for Agrarian Reform) }\end{array}$ \\
\hline MAQL & $\begin{array}{l}\text { Movimiento Armado Quintín Lame (Quintín Lame Armed } \\
\text { Movement) }\end{array}$ \\
\hline ILO & International Labor Organization \\
\hline NT & New Tribes (a missionary group) \\
\hline ONIC & $\begin{array}{l}\text { Organización Nacional Indígena de Colombia (National } \\
\text { Indigenous Organization of Colombia) }\end{array}$ \\
\hline SIL/WBT & Summer Institute of Linguistics/Wycliffe Bible Translators \\
\hline WHC & UNESCO World Heritage Center \\
\hline
\end{tabular}

\section{Glossary of Spanish Words and Phrases}

blanqueamiento

cabildo

capitanía

chuszua (Muiscubbun)

Derecho Mayor

guaquero

La Violencia

llanero

maloca

mesa de concertación

mestizaje

Pensamiento Propio

personería jurídica

rapé

resguardo

sede

taita

usos y costumbres

yajé whitening

council

small political unit headed by a capitán (headman)

temple

"Greater Right"

plunderer of archaeological sites

a period (1947-1967) of violent confrontations

between Liberal and Conservative parties

plainsman

multifamily longhouse

consultation roundtable

racial mixture, usually with reference to White and indigenous

"Our Own Thought"

legal personhood

potent tobacco snuff mixed with ashes of the

Yarumo tree (Pourouma cecropiaefolia)

communally owned indigenous reservation

headquarters

esteemed elder

uses and customs

Banisteriopsis caapi, a hallucinogenic infusion 